\title{
Improving access to medicines for mental disorders in low-resource settings: some achievements but still a long road ahead
}

\author{
C. Barbui* Editor and S. Chattherjee, Guest Editor
}

First published online 2 November 2015

A key component of any well-functioning health service, including mental health services, is the regular availability and appropriate use of medicines. In this Issue of Epidemiology and Psychiatric Sciences two interesting Editorials discuss some of the main barriers limiting access to medicines for mental disorders in low- and middle-income (LMI) settings and Asian countries, and suggest possible improvements. The topic is particularly relevant as up to one-third of people with mental disorders in LMI countries are unable to access essential medicines on a regular basis (World Health Organization, 2004).

A recent WHO Discussion Paper analysed some of the key barriers in relation to access to essential medicines for non-communicable diseases (World Health Organization, 2015). Several bottlenecks were identified which included: poor use of health technology assessment in decision-making for selection of medicines; poor health systems and supply chain governance; excessively strict regulation, as in the case of access to some controlled medicines; suboptimal utilisation of generic policies; high mark-ups and taxes; high prices due to existing intellectual property rights for medicines still under patent; poor implementation and use of standard treatment guidelines; poor acceptability by patients leading to lack of adherence; weak civil society participatory structures for advocacy and accountability measures for non-communicable diseases programmes. Challenges specific for LMI settings include inadequate financing of medicines, inappropriate selection of medicines, interrupted supply chains and high pricing (Bigdeli et al. 2013).

Challenges specific for mental disorders have also been identified (World Health Organization, 2005) and extensively discussed during a recent workshop organised by the Institute of Medicine Forum on

* Address for correspondence: Professor C. Barbui, Department of Public Health and Community Medicine, Section of Psychiatry, Ospedale Policlinico 'G.B. Rossi', Piazzale L.A. Scuro 10, 37134 Verona, Italy.

(Email: corrado.barbui@univr.it)
Neuroscience and Nervous System Disorders and the Forum on Health and Nutrition of the Uganda National Academy of Sciences (Pankevich et al. 2014).

As reported during the workshop, essential prerequisites for improving access to and rational use of psychotropic medicines are an adequate budget expenditure for mental disorders and the availability of services and trained personnel with the capacity to treat mental disorders. At a global level, it has been estimated that the mean expenditure for mental disorders is slightly less than $3 \%$ of the total health budget, and country income level does not fully account for the lower levels of funding for mental disorders (Pankevich et al. 2014). Low funding does not allow a proper development of services. In some African countries, for example, the number of outpatient health care services with the ability to treat mental disorders is low, which is a major infrastructural barrier to enhanced access and use of medicines (Pankevich et al. 2014).

In addition to infrastructural barriers, the following aspects have been identified as particularly relevant barriers for accessing medicines for mental disorders:

- In most LMI countries, the lack of accessible and affordable mental health services and the shortage of trained care providers is the most obvious challenge to making medication for mental disorders more widely available; in the absence of a system to deliver services, it is difficult to provide for medications widely and safely.

- Poor recognition of mental disorders by patients, families and health care professionals, and limited help-seeking behaviour due to stigma, discrimination or other socio-cultural factors, which decrease demand for medicines for mental disorders (Ensor \& Cooper, 2004; Jacobs et al. 2012). This is particularly important in many LMI countries where a variety of explanatory models are used to define mental disorders and where mental services are mostly available from institutional settings.

- Poor acceptability of psychotropic medicines as a remedy for 'behavioural abnormalities' that might 
not be conceptualised as mental health problems, together with the perceived low quality of health services and medicines.

- The long-term nature of many severe mental disorders, which has particular implications for access to services. Costs to patients and families may be unaffordable, as expenses are not only linked with an individual treatment or service, but also with the treatment having to be repeated over long periods. Related to this, the geographical location of communities and health services may be responsible for additional transportation cost and loss in working days, as medicines for mental disorders may be available only in selected secondary and tertiary health facilities.

- Poor adherence to treatment regimes, especially when difficulties with insight and cognitive functioning are present. As a consequence, treatment adherence is lower among patients with mental disorders than among patients with physical disorders (World Health Organization, 2005), and this is another challenging barrier specifically related to appropriate use.

- Lack of a reliable supply chain, as low level of current use of medicines for mental disorders, often related to the barriers reported above, may lead many supply chain actors to believe the true demand is low.

- High acquisition costs. Although most essential medicines for mental disorders are inherently cheap (at the point of production), a combination of import tariffs, sales taxes, mark-ups and other charges raises the cost to end-users. In populations with no financial protection or health insurance, out-of-pocket spending may be unaffordable.

- Strict regulations for some controlled medicines. This may apply to buprenorphine, diazepam and other benzodiazepines, as reported in the 25th edition of the Green List of Psychotropic Substances under International Control (International Narcotics Control Board, 2014). Methadone, included in the 52nd Edition of the Yellow List of narcotic drugs under international control, may also be subject to restrictions in access (International Narcotics Control Board, 2013). These medicines may be subject to specific storage requirements, and are often labelled as specialist drugs, which may mean that primary health care workers cannot prescribe them, dramatically decreasing their availability.

- Difficulties in the selection process. Selection of essential medicines for mental disorders may be particularly problematic, as the evidence base is in some cases of limited quality and difficult to interpret. Studies typically enrol small samples of highly selected patients shortly followed and assessed with outcome measures that are seldom used in practice. In addition, the cost-effectiveness of most medicines is not very well established, especially for newly developed medicines, which are often significantly more expensive than old medicines.

In order to overcome these barriers, action is required. A first action should be the implementation of legislations and policies promoting adequate access to essential psychotropic medicines. Legislations should ensure that appropriate pharmaceuticals are available at all times in mental health care delivery system. The products should be of acceptable quality, safe and efficacious, and not merely available, but also distributed and appropriately used by trained staff. Access to safe and cost-effective psychotropic medicines should also be an integral part of a mental health policy (World Health Organization, 2005).

Access to medicines would then depend on which medicines are selected for inclusion on a national essential medicines list, and whether they are available, affordable and appropriately used (Bigdeli et al. 2014). As reported in the two Editorials in this Issue of Epidemiology and Psychiatric Sciences, despite some remarkable achievements there is still a long road ahead for all the actors involved, including policy makers, researchers, health care professionals, service users, families and the wider society in making psychotropic medicines available more widely within health systems.

\section{Acknowledgements}

None.

\section{Financial Support}

This research received no specific grant from any funding agency, commercial or not-for-profit sectors.

\section{Conflict of Interest}

None.

\section{References}

Bigdeli M, Jacobs B, Tomson G, Laing R, Ghaffar A, Dujardin B (2013). Access to medicines from a health system perspective. Health Policy and Planning 28, 692-704.

Bigdeli M, Peters DH, Wagner A (2014). Medicines in Health Systems: Advancing Access, Affordability and Appropriate Use. World Health Organization: Geneva.

Ensor T, Cooper S (2004). Overcoming barriers to health service access: influencing the demand side. Health Policy and Planning 19, 69-79.

International Narcotics Control Board (2013). List of narcotic drugs under international control. Available at: https:// 
www.incb.org/documents/Narcotic-Drugs/Yellow_List/ NAR_2011_YellowList_50edition_EN.pdf

International Narcotics Control Board (2014). List of psychotropic substances under international control. Available at: https://www.incb.org/documents/ Psychotropics/green_lists/Green_list_ENG_2014_85222_ GHB.pdf

Jacobs B, Bigdeli M, Annear PL, Van DW (2012). Addressing access barriers to health services: an analytical framework for selecting appropriate interventions in low-income Asian countries. Health Policy and Planning 27, 288-300.

Pankevich D, Posey Norris S, Wizemann T, Altevogt B (2014). Improving Access to Essential Medicines for Mental,
Neurological, and Substance Use Disorders in Sub-Saharan Africa: Workshop Summary. Institute of Medicine. The National Academies Press: Washington, DC.

World Health Organization (2004). Equitable Access to Essential Medicines: a Framework for Collective Action. World Health Organization: Geneva.

World Health Organization (2005). Improving Access and use of Psychotropic Medicines. World Health Organization: Geneva.

World Health Organization (2015). Essential Medicines and Basic Health Technologies for Non-Communicable diseases: Towards a set of Actions to Improve Equitable Access in Member States. World Health Organization: Geneva. 